J. D. Bowman, W. J. Swiatecki and C. F. Tsang

July 1973

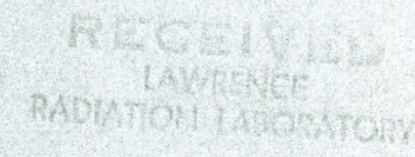

Prepared for the U.S. Atomic Energy Commission under Contract W-7405-ENG-48

TWO-WEEK LOAN COPY

This is a Library Circulating Copy

which may be borrowed for two weeks.

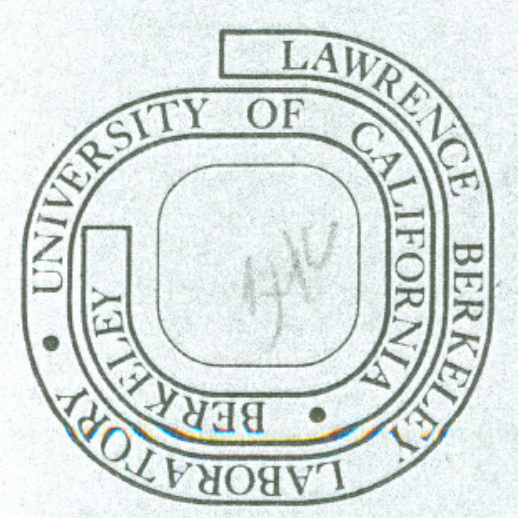
For a personal retention copy, call Tech. Info. Division, Ext. 5545 


\section{ABRASION AND ABLATION OF HEAVY IONS}

J. D. Bowman, W. J. Swiatecki, and C. F. Tsang

Lawrence Berkeley Laboratory

University of Califormia

Berkeley, California 94720

\section{July 1973}

We were stimulated by the first few seminars in this series to explore some simple interpretations of the Bevatron Heavy Ion experiment. We have spent a few weeks working out the consequences of an "abrasion-ablation" theory of these data and we are currently going on with this interpretation. New ideas are being injected every few days and this is a progress report on a fluid situation. Feedback from the audience will be particularly welcome.

The data we have concentrated on are those of Heckman, Iindstrom, Greiner, and Bieser, written up in a recent preprint. Let me first remind you of these data.

A beam of ${ }^{16} 0$ with $2.1 \mathrm{GeV} /$ nucleon is passed through a target and the cross-section measured for

$\begin{array}{llcc}160 & & \frac{\Delta z \text { los }}{\text { I6.g. for Pb target }} \\ \rightarrow & \text { Nitrogen } & 1 & 323 \mathrm{mb} \\ \rightarrow & \text { Carbon } & 2 & 258 \mathrm{mb} \\ \rightarrow & \text { Noron } & 3 & 123 \mathrm{mb} \\ & & 1,2,3, \ldots & 3100 \mathrm{mb}\end{array}$

the conversion of ${ }^{16} 0$ into various secondary beams corresponding to a loss of $1,2,3$ protons, and also the total for removing 160 from the beam.

The range of targets used was $\mathrm{H}, \mathrm{C}, \mathrm{S}, \mathrm{Cu}, \mathrm{Pb}$. In addition to ${ }^{16} \mathrm{O}$ beams, ${ }^{12} \mathrm{C}$ and ${ }^{4} \mathrm{He}$ beams were also studied. In other experiments the isotopic

* Berkeley Relativistic Heavy Ion Summer Study (1973). LBL internal report
Bev-3019 (July 23, 1973). 
composition of the emerging secondary beams and other fine details were analyzed but this is not discussed in the preprint and we have not gotten to that problem in our studies so far.

The dependence of the cross-section on target mass shows a general increase with A (as an example, see Fig. 1).

\section{Method of Calculation}

An obvious question arises: can these data be understood in terms of a shearing off of a piece of the 160 as it zooms through the target material? For example what cross-sections does one predict if one assumes nuclei to be sharp spheres with an equivalent sharp radius of $r_{0} A^{l / 3}\left(r_{0} \approx 1.2 F\right)$, and the 160 to be such a sharp sphere (radius $R_{l}$ ) which gets a piece gouged out of it every time it encounters a $\mathrm{Pb}$ target nucleus (radius $\mathrm{R}_{2}$ )? The calculation is of course rather trivial. We want to calculate the relative volume $\frac{\Delta Z}{Z}$ of the oxygen swept out by the $\mathrm{Pb}-$-the gometrical problem of the volume of intersection of a cylinder of radius $R_{2}$ with a sphere of radius $R_{1}$. The answer will be a function of the impact parameter b--the distance between the line of motion of the ${ }^{16} \mathrm{O}$ and the center of the $\mathrm{Pb}$ target nucleus. One can then ask: for what range of impact parameters $b_{\text {lower }}<b<b_{\text {upper }}$ does one shear off, say, one proton $\left(\frac{\Delta Z}{Z}=\frac{l}{8}\right.$ ) (more than $\frac{1}{2}$, less than $1 \frac{1}{2}$ ). The impact parameters falling in this range correspond then to Abrading $\frac{7}{\overline{8}}$ of the oxygen mass and on the average ending up with a nitrogen nucleus.

Before comparing the cross-section so obtained with the experimental $323 \mathrm{mb}$ we have to remember one thing. The nitrogen nucleus after the abrasion looks very funny, with a concave cylinarical surface where there used to be a 
convex spherical surface. Its surface energy is not what a healthy ground-state configuration of nitrogen would like, but quite a few $\mathrm{MeV}$ higher. This extra energy will frequently be dissipated by the emission (evaporation) of neutrons, protons, or $\alpha$-particles, in times of $10^{-17,-18} \mathrm{sec}$. So what is observed in the detector may be sometimes carbon and not nitrogen after all. In other words in a given collision we have to allow for a further loss of material out of the initial ${ }^{16} 0$, due to conversion of excitation energy (resulting from the collision) into evaporated particles. A good word to describe this is Ablation. So we have the picture

$\begin{array}{ll}\text { Abrasion: } & \Delta \mathrm{Z}_{\mathrm{Abr}}(\mathrm{b}) \quad \text { (shearing off of volume) } \\ \text { Ablation: } & \Delta \mathrm{Z}_{\mathrm{Abl}} \text { (b) } \\ \Delta \mathrm{Z}(\mathrm{b})=\Delta \mathrm{Z}_{\mathrm{Abr}}+\Delta \mathrm{Z}_{\mathrm{Abl}}\end{array}$

How do we estimate $\Delta \mathrm{Z}_{\mathrm{AbI}}$ ?

We said: from the geometry of the funny-looking nitrogen we can calculate its excess surface area and so its excess surface energy, say $\Delta E_{S}$. (The surface energy of nuclei is known as about $1 \mathrm{MeV} / \mathrm{F} .^{2}$ ) It takes about $10 \mathrm{MeV}$ to evaporate a nucleon, so the average number of protons evaporated will be about

$$
\Delta \mathrm{Z}_{\mathrm{AbI}} \sim \frac{\Delta \mathrm{E}_{\mathrm{S}}}{20 \mathrm{MeV}}
$$

So the final number of protons lost is

$$
\Delta \mathrm{z}(\mathrm{b})=\Delta \mathrm{z}_{\mathrm{Abr}}(\mathrm{b})+\Delta \mathrm{z}_{\mathrm{AbI}}(\mathrm{b})
$$


where the first part follows from the geometry of the volume sheared off and $\Delta Z_{A b l}$ from the geometry of the area sheared off.

\section{Calculated Results and Discussion}

We can now calculate the range of impact parameters $b_{\text {lower }}<b<b_{\text {upper }}$ for removing say one proton from the ${ }^{16} 0$ and then calculate the cross section for producing nitrogen from oxygen by

$$
\sigma(0 \rightarrow N)=\pi\left(b_{\text {upper }}^{2}-b_{\text {lower }}^{2}\right)
$$

Nothing is adjustable in such a calculation and one wonders what will come out, $10 \mathrm{mb}, 100 \mathrm{mb}$, $1000 \mathrm{mb}$ to compare with the $323 \mathrm{mb}$ for the case of $\mathrm{Pb}$ as target. How will the answer depend on target mass?

Well, the answer we got was $419 \mathrm{mb}$ instead of $323 \mathrm{mb}$. The dependence on mass was as shown. There is a general increase with $\mathrm{A}^{1 / 3}$, but with a discrepancy of about a factor of two.

Using our calculated function $\Delta Z(b)$ we could also determine the range of impact parameters for removing 2 and 3 protons (as well as one) and thus calculate all three cross-sections

$$
\begin{array}{ll}
\sigma(0 \rightarrow N)=\pi\left(b_{0.5}^{2}-b_{1.5}^{2}\right) & \frac{\text { Calc. }}{419} \\
\sigma(0 \rightarrow C)=\pi\left(b_{1.5}^{2}-b_{2.5}^{2}\right) & \frac{\text { Expt. }}{(313)} \\
\sigma(0 \rightarrow B)=\pi\left(b_{2.5}^{2}-b_{3.5}^{2}\right) & 286
\end{array}
$$

where $b_{1.5}$ is the value of $b$ at which $\Delta z=1.5$, and $b_{2.5}$ is the value of $b$ at which $\Delta \mathrm{z}=2.5$, so that for $\mathrm{b}_{2.5}<\mathrm{b}<\mathrm{b}_{1.5}$ two protons are most often removed. Note also that according to the logic we are using, $\pi b_{0.5}^{2}=\sigma_{\text {Total }}$ 
gives the cross section for removing 1 or 2 or $3 \ldots .$. charges i.e. for removing 160 out of the beam altogether. This has the measured value of $3100 \mathrm{mb}$ with $\mathrm{Pb}$ as target, to be compared with $\pi \mathrm{b}_{0.5}^{2}$ of $2700 \mathrm{mb}$ (calculation $20 \%$ too $10 \mathrm{w}$ ). The table below summarizes our estimates at this stage of the game (using $r_{0}=1.2$ ):

\begin{tabular}{|c|c|c|c|c|c|}
\hline & & & Target & & \\
\hline$\sigma_{0} \rightarrow \mathbb{N}$ & $\begin{array}{c}\underline{\mathrm{H}} \\
64 \pm 11 \% \\
(121)\end{array}$ & $\begin{array}{c}\frac{\mathrm{c}}{104 \pm 6 \%} \\
(208)\end{array}$ & $\begin{array}{c}\frac{\mathrm{S}}{121} \pm 8 \% \\
(263)\end{array}$ & $\begin{array}{c}\frac{\mathrm{Cu}}{164 \pm 7 \%} \\
\text { (299) }\end{array}$ & $\begin{array}{c}\frac{\mathrm{Pb}}{323 \pm 7 \%} \\
(419)\end{array}$ \\
\hline $0 \rightarrow C$ & $\begin{array}{c}58 \pm 14 \% \\
(67)\end{array}$ & $\begin{array}{c}130 \pm 5 \% \\
(130)\end{array}$ & $\begin{array}{c}139 \pm 8 \% \\
(181)\end{array}$ & $\begin{array}{c}190 \pm 6 \% \\
(219)\end{array}$ & $\begin{array}{c}258 \pm 7 \% \\
(286)\end{array}$ \\
\hline$+B$ & $\begin{array}{c}34 \pm 17 \% \\
(45)\end{array}$ & $\begin{array}{c}61 \pm 8 \% \\
(97)\end{array}$ & $\begin{array}{c}73 \pm 10 \% \\
(142)\end{array}$ & $\begin{array}{c}86 \pm 9 \% \\
(175)\end{array}$ & $\begin{array}{c}123 \pm 11 \% \\
(239)\end{array}$ \\
\hline $\begin{array}{c}0 \rightarrow \text { not } 0 \\
\sigma_{\text {Tot }}\end{array}$ & $\begin{array}{r}315 \pm 7 \% \\
(343)\end{array}$ & $\begin{array}{c}935 \pm 2 \% \\
(743)\end{array}$ & $\begin{array}{c}1310 \pm 4 \% \\
(1100)\end{array}$ & $\begin{array}{c}1820 \pm 2 \% \\
(1500)\end{array}$ & $\begin{array}{c}3100 \pm 2 \% \\
(2700)\end{array}$ \\
\hline
\end{tabular}

Numbers in brackets give the calculated cross-sections in mb according to "clean cut" version of abrasion/ablation theory (sharp surfaces). The following points are observed:

1) Calculated cross sections in the right ball-park, BUT:

2) Too high by a factor of about 2 for $O \rightarrow N$, $O \rightarrow B$, not so much for $0 \rightarrow C$ (an odd-even effect which might be associated with the odd-even variations of nucleon binding energies which would influence the evaporation).

3) Total cross-sections too low.

A comparison of the exp. and calculated cross-sections as functions of target mass is shown in Figs. 1-4.

From our point of view it is not very useful to replot these on a log-log scale, but Heckman et al. have such a plot and some people are 
interested in the resulting slopes, when one tries to write e.g.

$$
\sigma(O \rightarrow N)=\text { const } A_{\text {target }}^{S}
$$

Figure 5 shows a comparison of the experimental points, some of which roughly follow an exponent $s \approx 0.26$ with the average calculated trend, which has a slope $s \approx 0.29$. From the point of view of the abrasion/ablation picture the expected behavior is not an exact straight line in a $\log \sigma$ vs $\log \mathrm{A}_{2}$ plot and such plots are of limited usefulness to us. Instead one might try to relate the fact that the slopes that come out are somewhat less than $\frac{1}{3}$ to the observation that the relevant collisions are grazing collisions, with the impact parameter $\mathrm{b}$ approximately the sum of the radii

$$
b \sim R_{1}+R_{2}-\bar{l}=r_{0}\left(A_{2}^{I / 3}+A_{e f f}^{I / 3}\right)
$$

where $\ell$ is a mean interpenetration depth and

$$
A_{\text {eff }}^{I / 3}=\frac{R_{I}-\bar{l}}{r_{0}} \text {, a number }<A_{I}^{I / 3} \text {. }
$$

If we take

$$
\sigma=k\left(A_{2}^{I / 3}+A_{e f f}^{I / 3}\right)
$$

then

$$
s=\frac{d(\ln \sigma)}{d\left(\ln A_{2}\right)}=\frac{1}{3} \frac{A_{2}^{I / 3}}{A_{2}^{1 / 3}+A_{e f f}^{I / 3}}
$$

For very large $A_{2}, s \rightarrow 0.33$. For finite $A_{2} s$ drops below $\frac{1}{3}$ because of $A_{\text {eff }}^{1 / 3}$. Inserting typical values of $R_{\text {eff }}\left(A_{\text {eff }}^{I / 3}\right)$ one gets slopes like these 


$\begin{array}{llll}\underline{\Delta z=1} & \underline{\Delta \mathrm{z}=2} & \underline{\Delta \mathrm{z}=3} & \\ 0.266 & 0.287 & 0.307 & \mathrm{~A}_{2}=208 \\ 0.202 & 0.235 & 0.272 & \mathrm{~A}_{2}=12\end{array}$

(The slopes are steeper for $\Delta z=3$ because $R_{\text {eff }}$ is less for many particles sheared off.)

\section{Further Discussions}

Rather than discussing the log-log slopes we find it more informative to make plots that compare the theoretical abrasion-ablation curves with experiment.

Let us first show you two calculated abrasion-ablation curves

as a function of the overlap depth $\ell$ in $\mathrm{fm}$, for ${ }^{208} \mathrm{~Pb}$ and $\mathrm{H}$

abrading ${ }^{16} 0$ (Figs. 6,7). Each curve is split into its two components

$\Delta \mathrm{z}_{\mathrm{abr}}$ and $\Delta \mathrm{z}_{\mathrm{abl}}$. The latter is very important for $\mathrm{H}$, less so for

$\mathrm{Pb}$. Intermediate targets show intermediate behavior. Scale is in fm. At

$l=2.9$ fm the edge of the knife goes through the center of the ${ }^{16} 0$. These

are the key curves of the theory and it is from intercepts dropped at the

$0.5,1.5,2.5,3.5$ levels that one calculates $\sigma_{\text {Tot }}, \sigma_{1}, \sigma_{2}, \sigma_{3}$.

Can we make a direct comparison of these curves with experiment? In

other words what experimental abrasion-ablation curves would give the exact experimental cross sections? The extraction of the experimental abrasion-ablation curves from the data is very easy:

$$
\begin{gathered}
\sigma_{\text {Tot }}=\pi\left(R_{1}+R_{2}-\ell_{0.5}\right)^{2} \\
\therefore l_{0.5}=R_{1}+R_{2}-\sqrt{\frac{\sigma_{\text {Tot }}}{\pi}}
\end{gathered}
$$




$$
\begin{aligned}
\sigma_{1} & =\pi\left(R_{1}+R_{2}-l_{0.5}\right)^{2}-\pi\left(R_{1}+R_{2}-l_{0.5}\right)^{2} \\
\therefore l_{1.5} & =R_{1}+R_{2}-\sqrt{\frac{\sigma_{\text {Tot }}-\sigma(1)}{\pi}} \\
l_{2.5} & =R_{1}+R_{2}-\sqrt{\frac{\sigma_{\text {Tot }}-\sigma_{1}-\sigma_{2}}{\pi}} \\
l_{3.5} & =R_{1}+R_{2}-\sqrt{\frac{\sigma_{\text {Tot }}-\sigma_{1}-\sigma_{2}-\sigma_{3}}{\pi}} \text { etc. }
\end{aligned}
$$

In this way we get point by point an experimental plot of the overlap or gouging depth at which $0.5,1.5,2.5,3.5$ protons are removed. The results are shown in Fig. 8. The position of the calculated abrasion-ablation curves (approximately the same for all targets) is indicated by the dashed curve. The experimental results are shown as solid curves (with $r_{0}=1.16$ ). We note the following:

1) Position of curves wrong by $1 \mathrm{fm}$, except for $H$.

2) Experimental curves much steeper--by a factor of two. (This is the factor of two that gives too high cross-sections).

3) All targets except $\mathrm{H}$ grouped fairly closely--i.e. experimental abrasion-ablation curve not very much dependent on target i.e. on the curvature of the knife that is doing the abrasion. Hydrogen is further in: this means that to do the same amount of damage to ${ }^{16} \mathrm{O}$ using $\mathrm{H}$ one has to cut deeper (by $l \mathrm{fm}$ ). This seems reasonable since $\mathrm{H}$ is a rather meager type of knife.

4) $\mathrm{Cu}, \mathrm{Pb}$ curves seem out of line, but only by $\frac{1}{10} \mathrm{~F}$.

5) Some points go to negative values. This is alright if we remember that zero is where effective sharp surfaces touch. Even for negative $l$ values, the tails of density distributions interact. 
We took a quick look at estimating the effect on the calculations of introducing diffusion in the nuclear surface and we are fairly certain that the effect will be to accentuate the discrepancy (the calculated curves will get washed out and have an even gentler slope, leading to higher partial cross sections).

Our feeling at the moment is that despite cross-section predictions that are generally in the right ball-park there is evidence for a very serious deficiency in the model examined so far. Take a penetration depth of $1 \mathrm{fm}$. Theory says about 0.6 of a proton should be lost, experiment (except H) says it is about $3-3 \frac{1}{2}$. This is a factor of 5 off. We think we are missing a very important piece of physics, perhaps a dominant component of the process.

\section{Dirty-Cut or Gangrenous-Bruise}

We think this is also what one arrives at by examining the physics of the collision. When a nucleon or even a collection of extremely energetic nucleons zips through ${ }^{16} 0$ one does not really expect a piece of the ${ }^{16} 0$ to be ripped off instantaneously. What one expects is the fast nucleon(s) to zip through, leaving behind recoiling target nucleons and $(\pi)$ mesons. The recoil velocities are smallish--50 - $100 \mathrm{MeV}$ we are told, so on a fast time scale nothing much happens at first. The region of overlap--the swiped region--far from having been swept clean is a region where energy and even rest mass (of the pions) has been deposited. Moreover the directions of the recoiling nucleons and pions are not well collimated forward--the partners of elastic $n-n$ collisions actually go mostly at angles close to $90^{\circ}$ to the beam. So what one has is a hot region--a bruise--with 50-100 MeV nucleons and pions radiating out. Those directed away from the ${ }^{16} 0$ will indeed escape, but others, perhaps a half--will irradiate the ${ }^{16} 0$, heat it up and make it lose more nucleons than one had thought. 
So our present tendency is to go away from a clean-cut abrasion to a dirty, gangrenous bruise that develops into a boil (and raises the patient's temperature). The end result is the loss of a leg from a bruised foot! To develop a theory of such a process there seems to be two elements necessary

I) A phenomenological theory of the deposition of mass, energy and momentum in the swiped region (the bruise). Perhaps the concept of a volume friction will be useful.

2) The dispersal of the bruise: partly by direct sublimation and partly by transfer of energy to the oxygen and subsequent evaporation.

In a formulation such a macroscopic theory we need help from people who are familiar with the angular and energy distribution of the microscopic elements--recoiling nucleons and mesons.

Elaboration

Figure 9 is an attempt to imagine what the result might be of carrying out a calculation in which two fast nuclei (comparable in size) collide and the energy (and momentum) dissipation is described by a friction term which is a function of the overlap of the two density distributions $\rho_{l}(\vec{r})$ and $\rho_{2}(\vec{r})$. For example one might try to describe the rate of energy dissipation (i.e. energy taken out of the collective kinetic energies and converted into internal excitations and meson production) by a term of the form

$$
\frac{d E}{d t}=-K \int \rho_{1}(\vec{r}) \rho_{2}(\vec{r}) f\left(\vec{v}_{12}^{2}\right) d{ }^{3} \vec{r}
$$

Here $f$ is some increasing function of the square of the relative velocity $\overrightarrow{\mathrm{v}}_{12}$ between the mass elements of the two fragments of the point $\vec{r}$ and $K$ is a friction or dissipation coefficient. 
Figure 9 shows a set of ten velocity (or rapidity) diagrams representing various possible results of such a collision as a function of the impact parameter and the magnitude of the friction coefficient $K$. If the impact parameter is greater than the contact value, or if $K=0$, there is essentially no interaction and the rapidity diagram shows two delta functions corresponding to the unperturbed projectile and target.

At the other extreme, if two comparable nuclei collide head on and the friction is large enough to soak up all the kinetic energy (i.e. the nuclei are brought to rest in the center of mass frame), one would find a single fireball centered in the midale of the rapidity plot, though there might be a large spread extending even to the edges of the rapidity diagram.

If $K$ is imagined decreased (but we still confine ourselves to a head-on collision) then the two nuclei will begin to pass through each other, but with reduced velocities. The single central hump in the rapidity diagram will then split into two humps, which move to the edges of the diagram as the friction goes to zero.

On the other hand if the friction is kept large but the impact parameter is increased, one expects the overlapping portions of the nuclei (the bruises) to be brought to rest but the non-overlapping portion to keep going with approximately their initial velocities. One would then see three humps in the rapidity diagram, two at the edges and one in the center. The area of the central hump representing the stopped overlapping region would go to zero as the impact parameter tends to the contact value. If, with an intermediate impact parameter, the friction is decreased then the overlapped portions of the nuclei will not be brought to rest but will pass through each other with diminished velocities. One then expects 
four humps as shown in the central one of the $3 \times 3$ set of pictures in Fig. 9 . As the friction coefficient $\mathrm{K}$ is decreased the two middle humps move out towards the edges. At some critical value of $\mathrm{K}$ there will in fact come a stage where the momentum deposited in the bruises by the frictional force is not large enough to tear them away and below that critical value only the side humps would be present. The critical friction at which this happens would be a function of the impact parameter, and the dotted line across Fig. 9 is meant to be an indication of the existence of such a critical locus.

The widths of all the humps are not intended to be to scale. Evaporation from the excited residues or bruises would be one factor contributing to the widths, but other dynamical or pre-equilibrium processes might be equally or more important.

\section{Acknowledgements}

This work was done under the auspices of the U. S. Atomic Energy Commission. 


\section{Figure Captions}

Fig. 1. Comparison of experimental and calculated cross-sections as a function of target mass for the conversion of ${ }^{16} 0$ to nitrogen isotopes.

Fig. 2. Same as Fig. 1, but for the conversion of ${ }^{16} 0$ to carbon isotopes.

Fig. 3. Same as Fig. 1, but for the conversion of ${ }^{16} 0$ to boron isotopes.

Fig. 4. Comparison of experimental and calculated total cross-sections.

Experimental points are indicated by open triangles and the calculated values are indicated by a smooth curve.

Fig. 5. Display of results in a log-log plot. Symbols are explained in the figure. Our calculated results are indicated by three thin straight lines, labelled $0^{16} \rightarrow \mathrm{B}, 0^{16} \rightarrow \mathrm{C}$, and $0^{16} \rightarrow \mathrm{N}$, respectively.

Fig. 6. Abrasion-ablation curves for ${ }^{16} 0$ on a hydrogen target. The number of protons loss from the ${ }^{16} 0$ nucleus is plotted against the inter-penetration depth $\ell$ in Fm.

Fig. 7. Same as Fig. 6, but for ${ }^{16} 0$ on ${ }^{208} \mathrm{~Pb}$ target.

Fig. 8. Experimentally deduced abrasion-ablation curves for ${ }^{16} 0$ on various target nuclei. The position of the calculated abrasion-ablation curves (approximately the same for all targets) is indicated by the dashed curve.

Fig. 9. Rapidity diagrams for various impact parameters and strengths of frictional coefficient. See text for detailed description. 


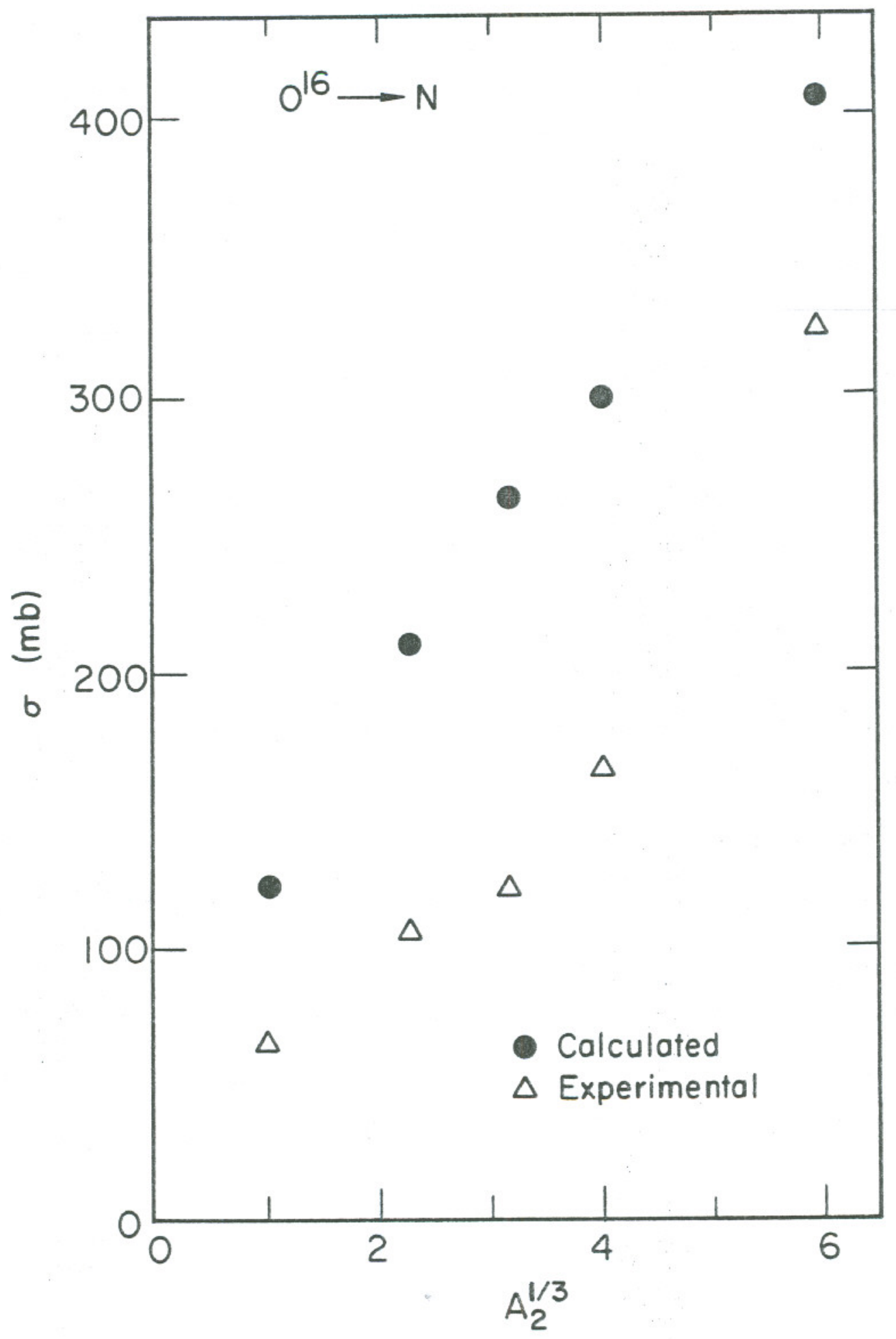

$X B L 737-3330$

Fig. 1. 


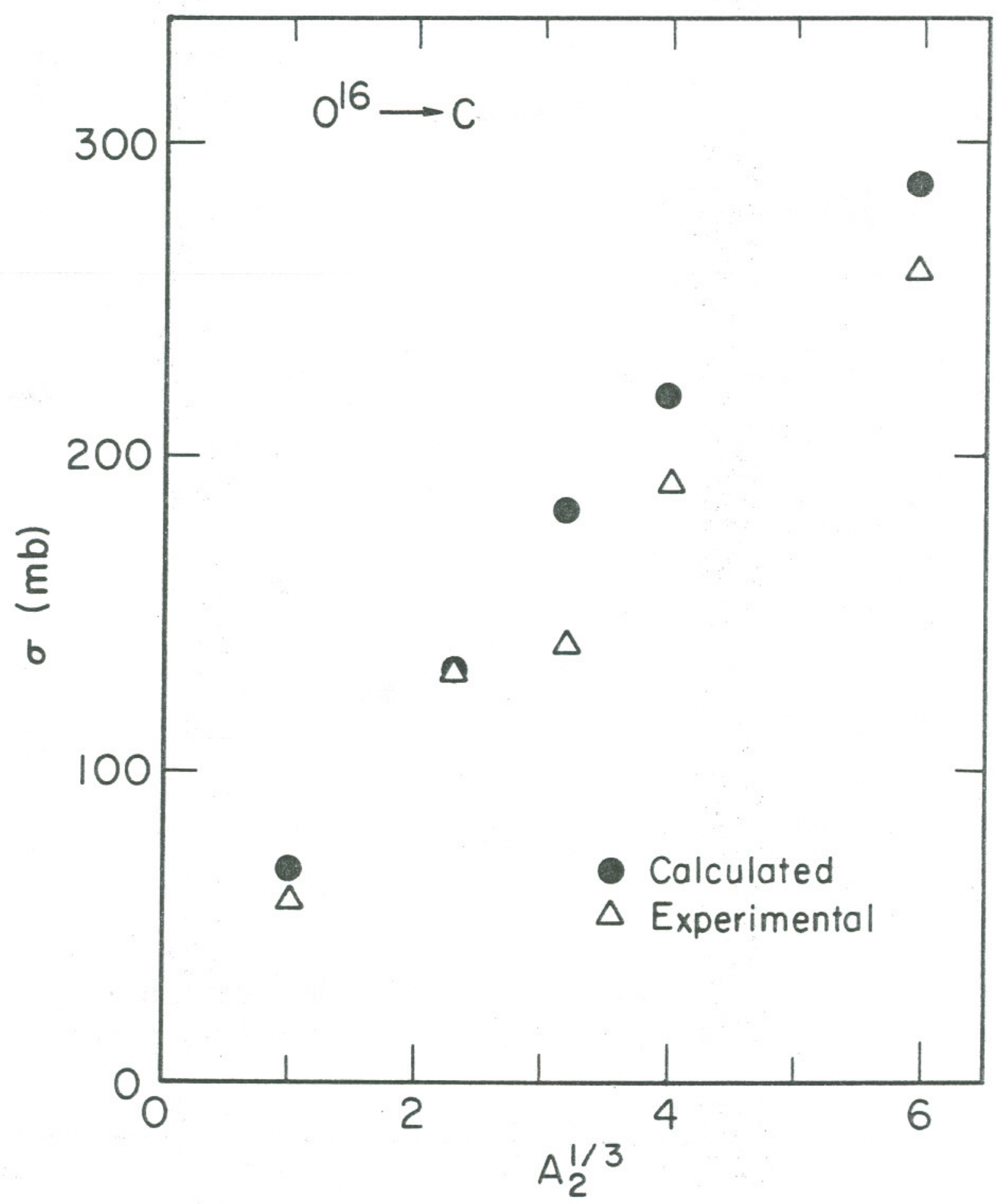

XBL $737-3329$

Fig. 2. 


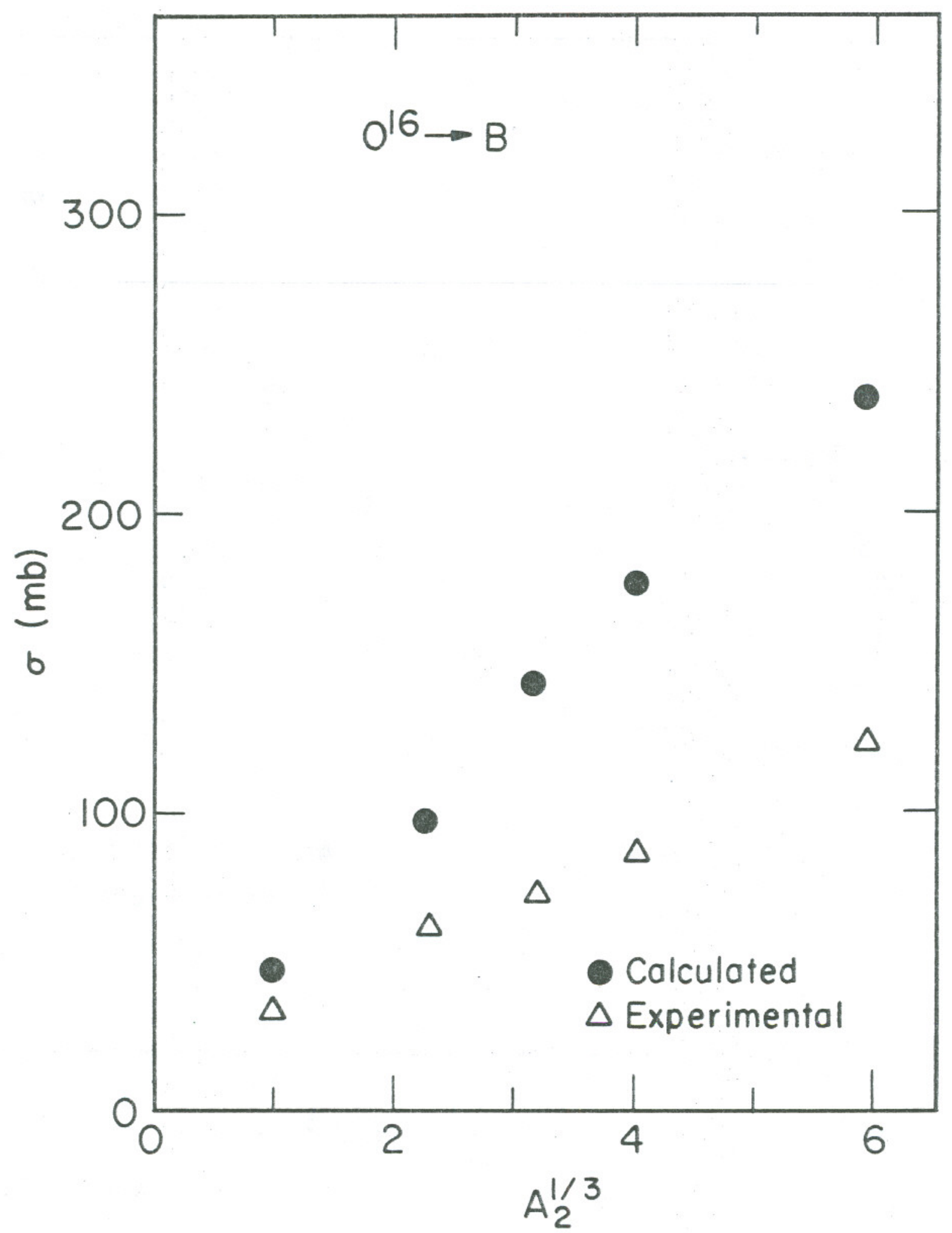

XBL $737-3328$

Fig. 3. 


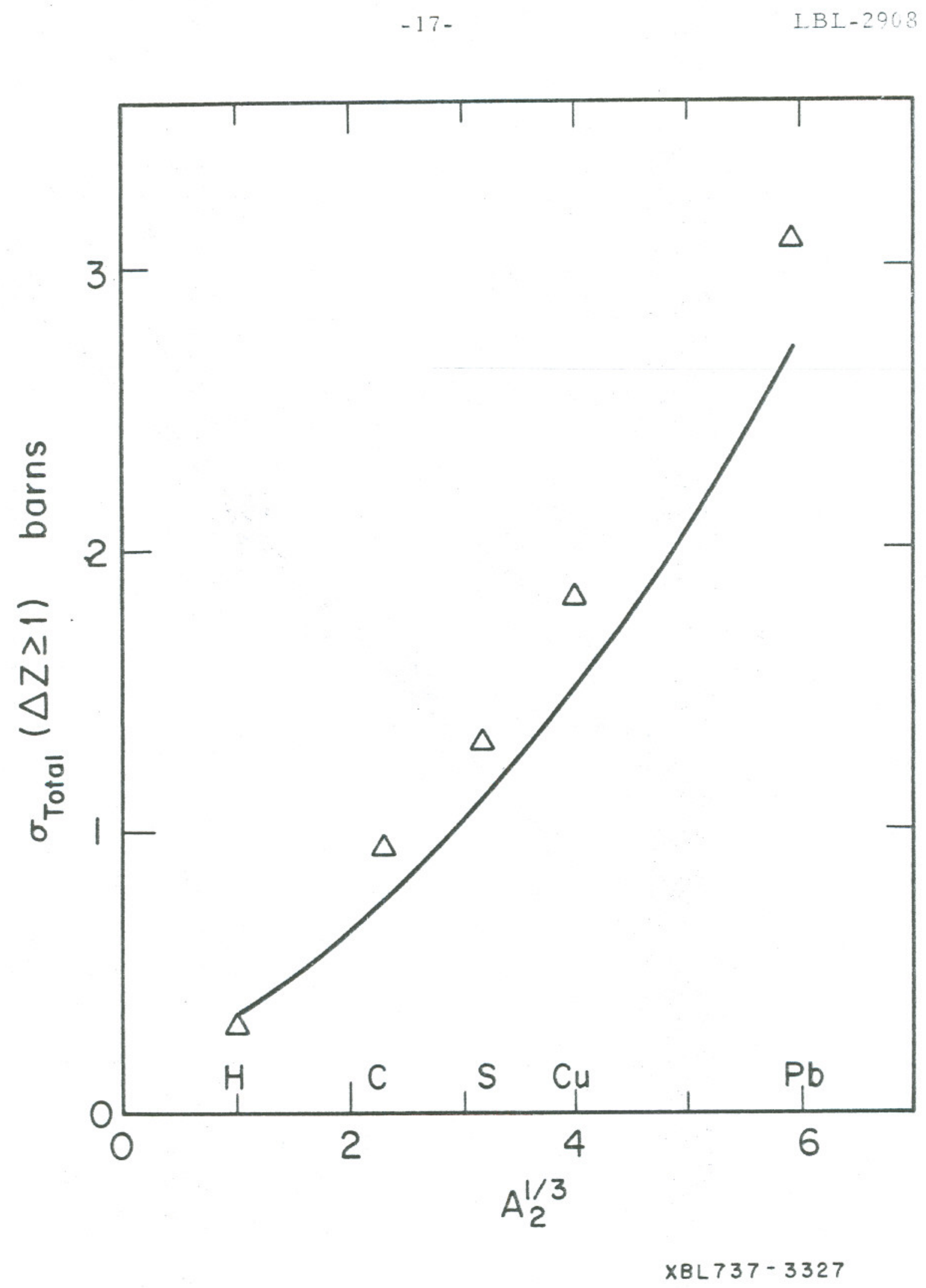

Fig. 4. 


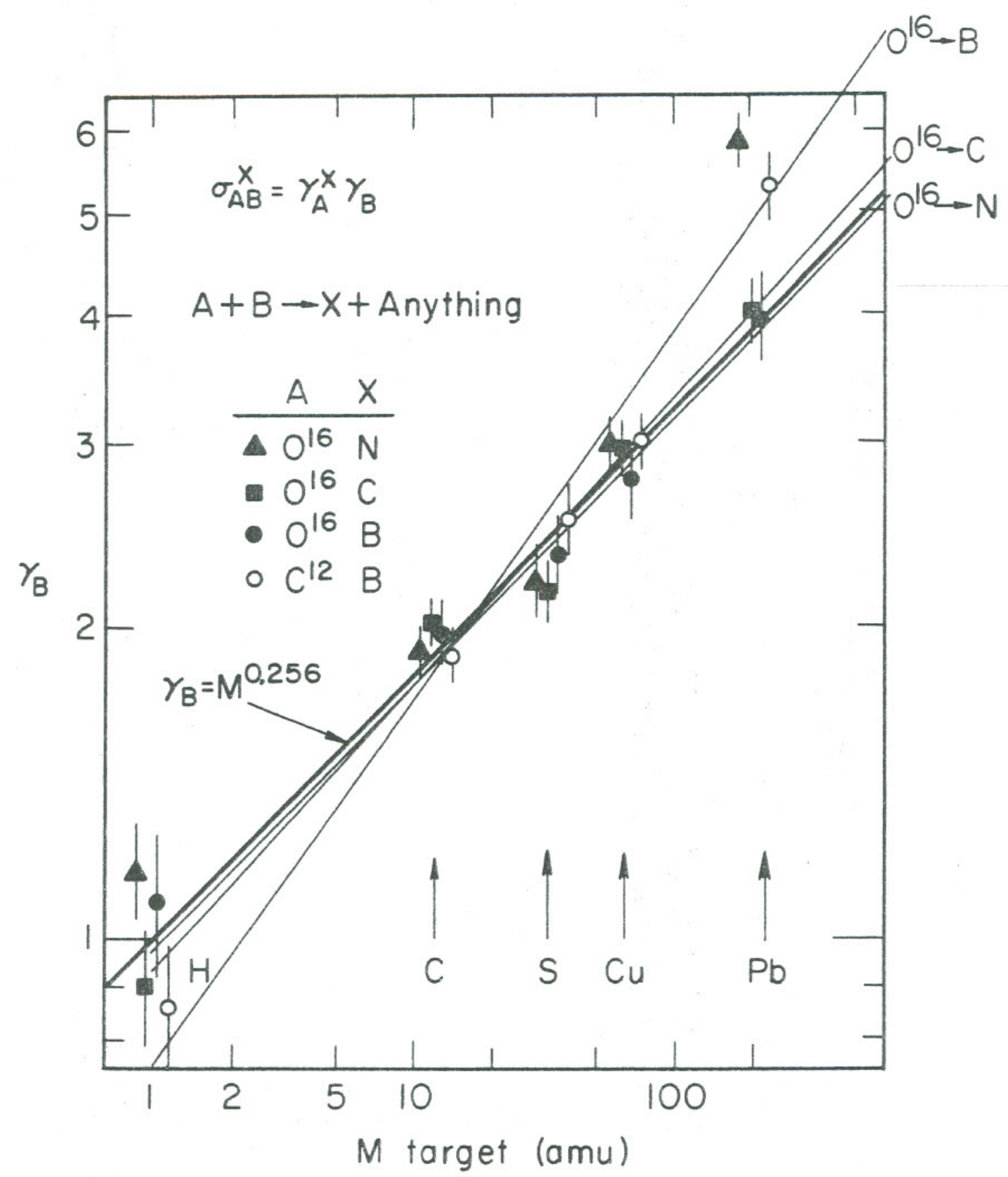

XBL $737-3331$

Fig. 5 . 


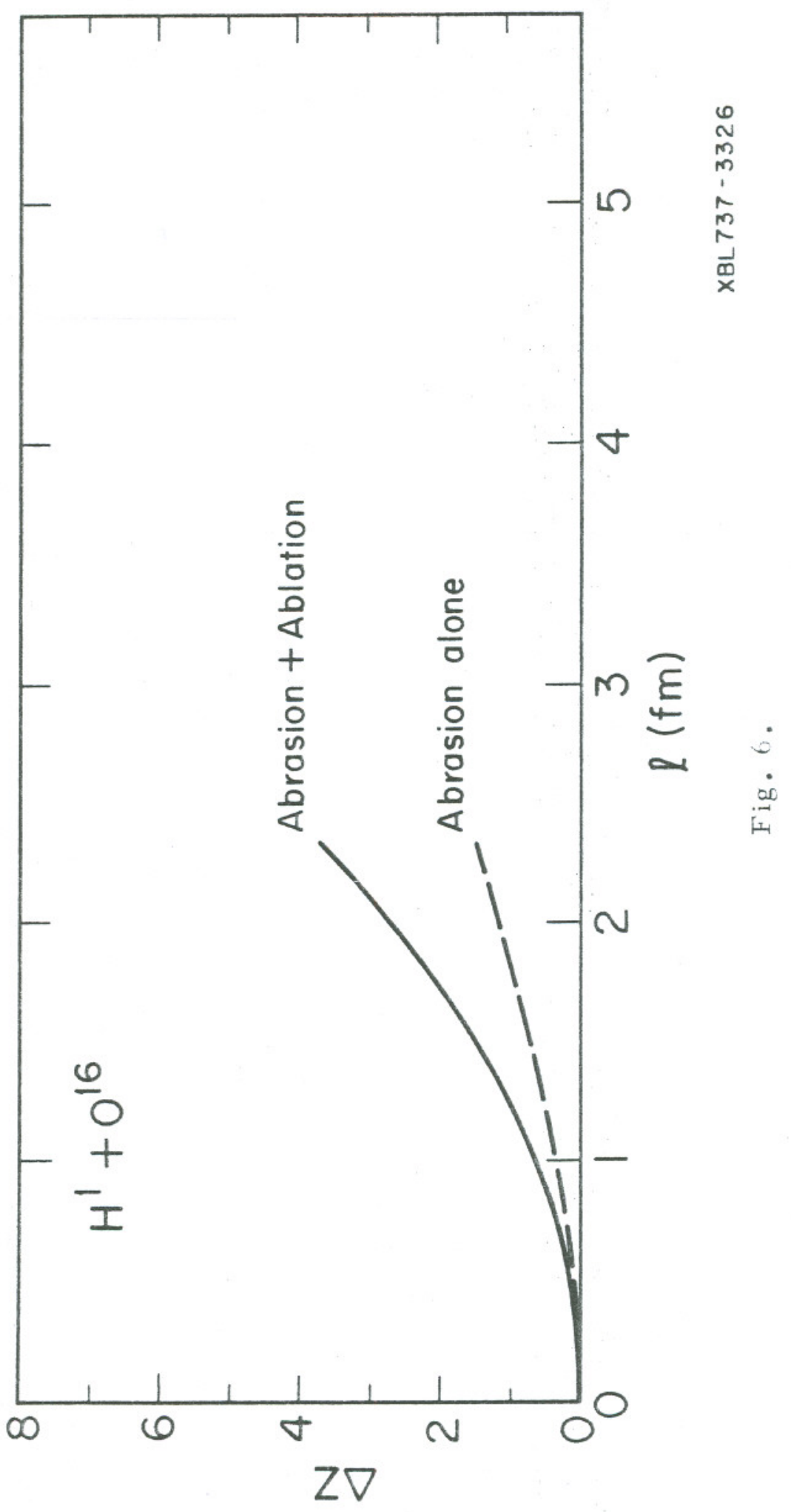




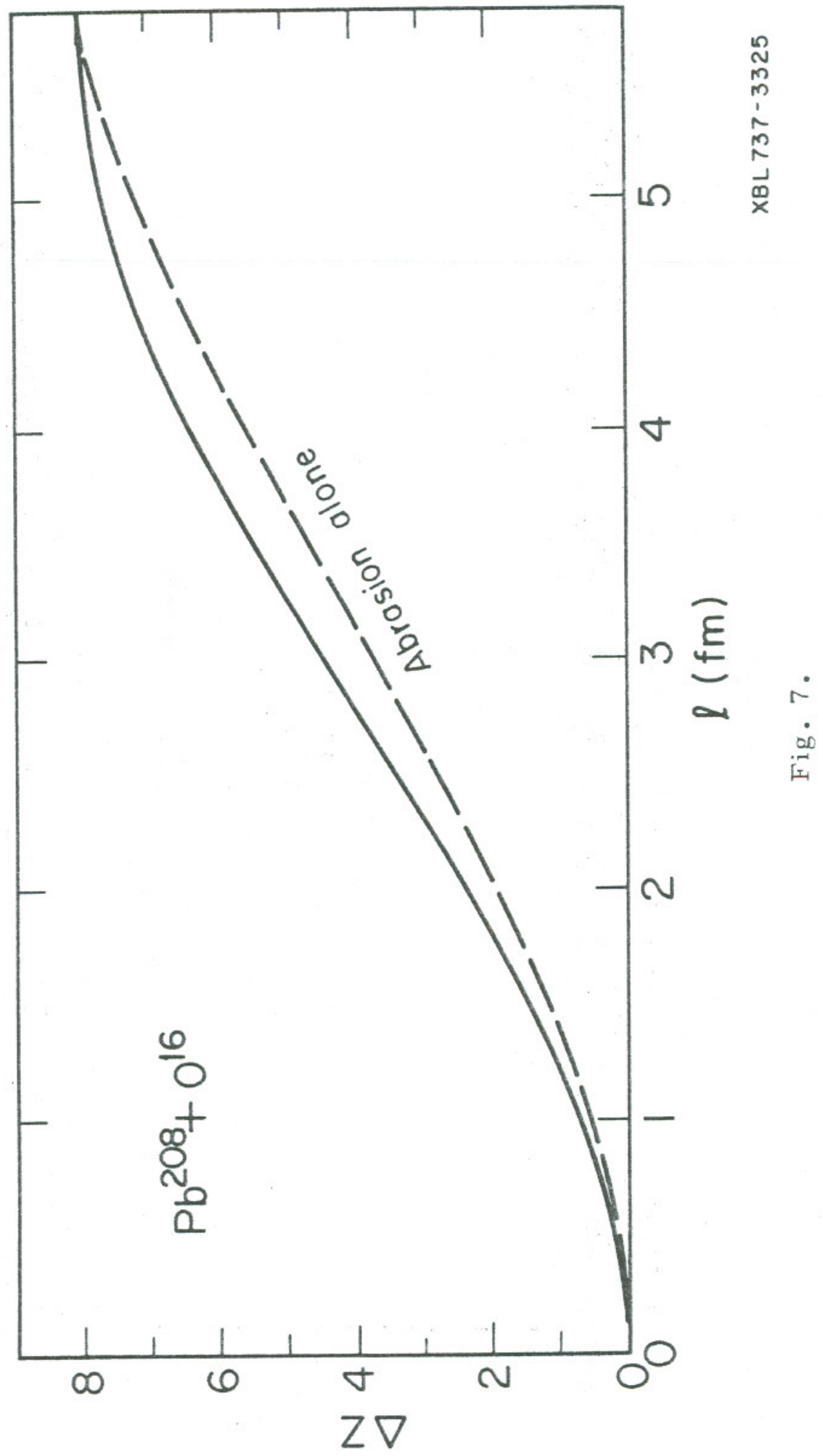




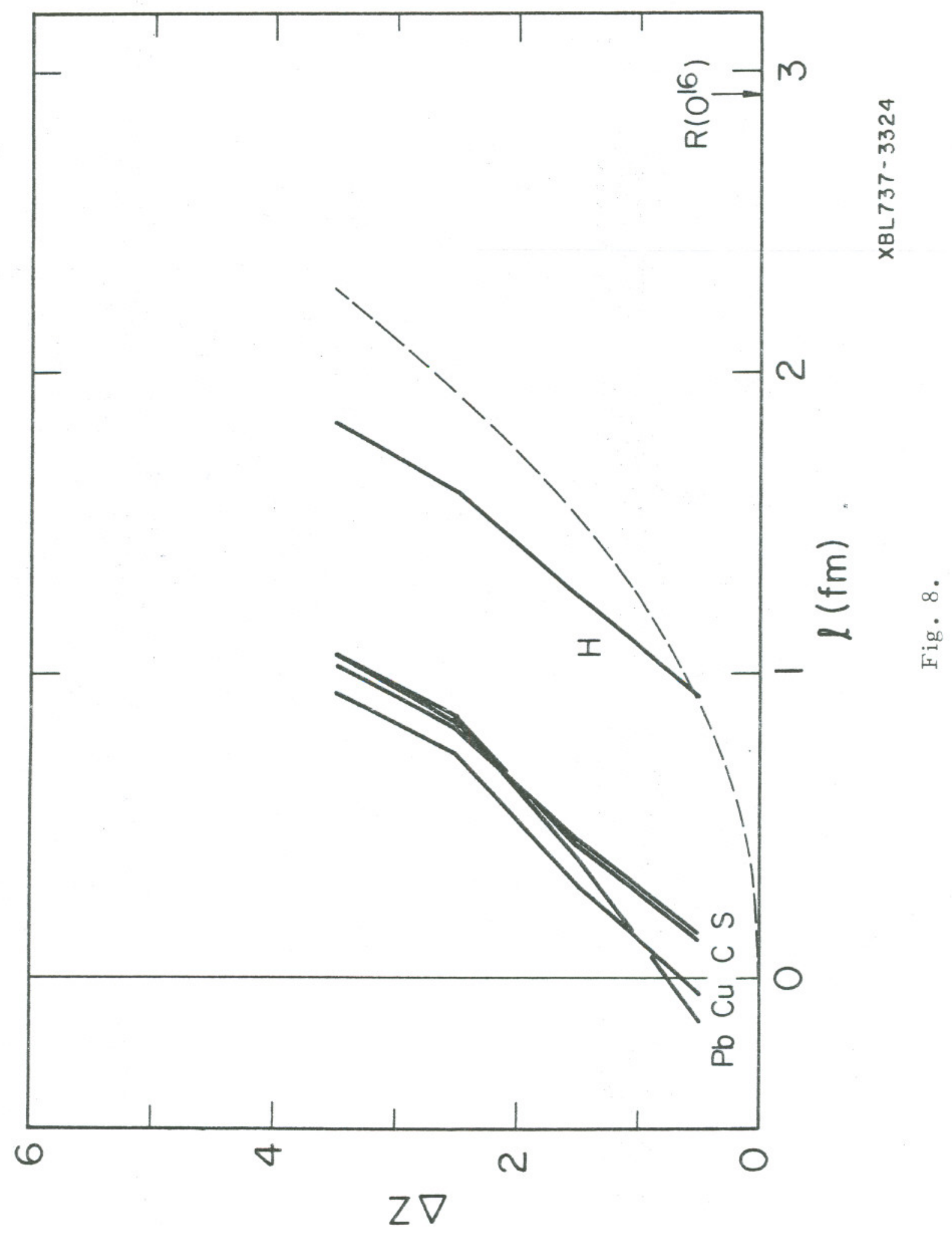




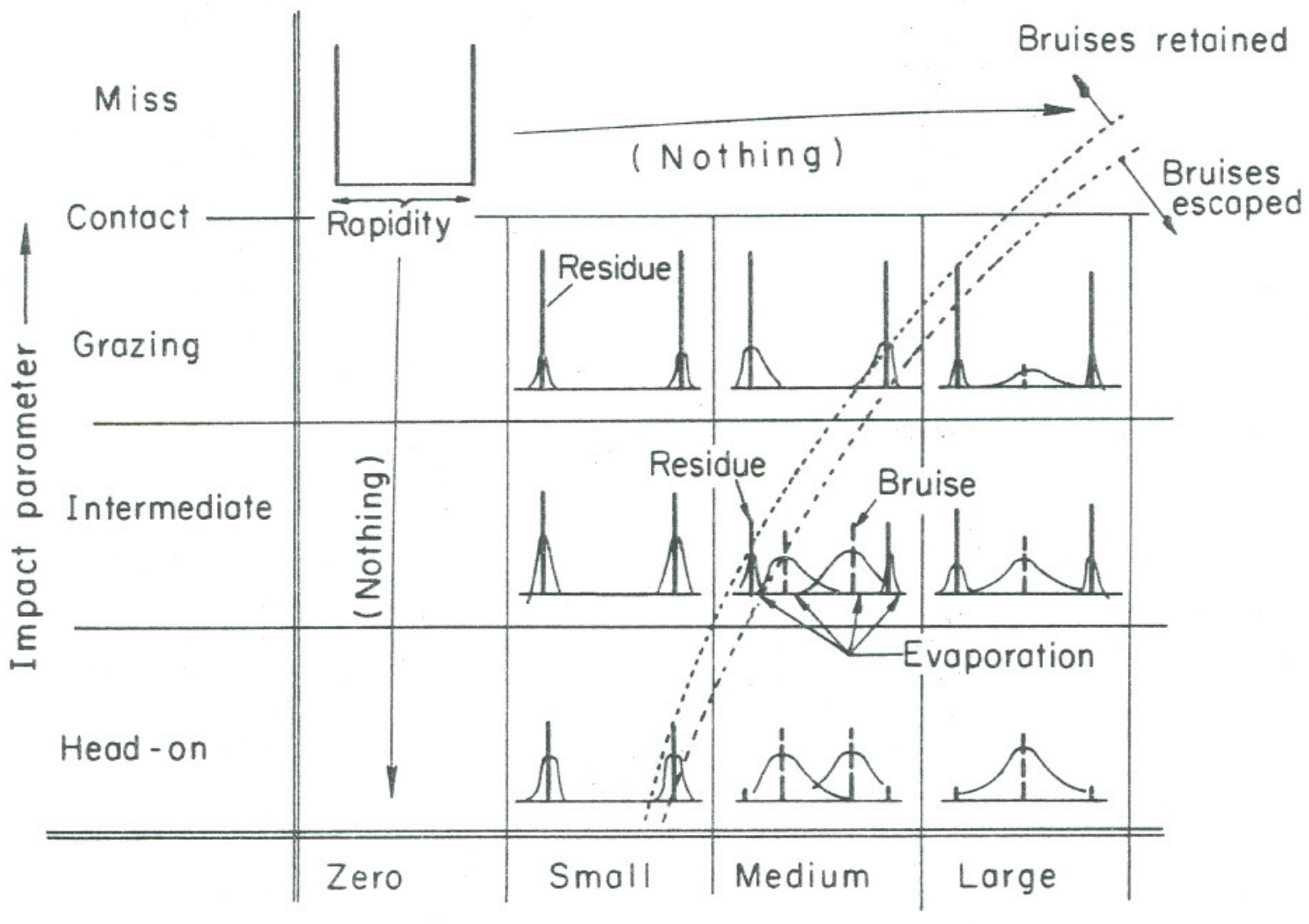

Friction coefficient $\mathrm{K} \longrightarrow$

Fig. 9. 


\section{LEGAL NOTICE}

This report was prepared as an account of work sponsored by the United States Government. Neither the United States nor the United States Atomic Energy Commission, nor any of their employees, nor any of their contractors, subcontractors, or their employees, makes any warranty, express or implied, or assumes any legal liability or responsibility for the accuracy, completeness or usefulness of any information, apparatus, product or process disclosed, or represents that its use would not infringe privately owned rights. 